achieved in $86.7 \%(13 / 15)$ of patients. Notably, one patient was approached via primary radial access, however resorted to a transfemoral approach; successful revascularization to mTICI 2 c was achieved.

As may be expected, in patients where radial access second intention, time to clot engagement was 91 minutes [IQR 51.5-125.5] $($ mean $=115.6 \pm 53.8)$ from initial attempts; however, from secondary transradial attempts, median time was 44 minutes [14.0-77.0] (mean $=52.7 \pm 46.3$ ).

The median time difference between initial puncture to radial access rescue therapy was 14 minutes [IQR 2-46.5] (mean $35.7 \pm 52.0)$. Success was achieved in 70.6\% (12/17) of cases in this cohort. Overall, successful revascularization was achieved in $78.1 \%(25 / 32)$ of patients; mTICI scores were not available in 2 patients. There were 4 peri-procedural complications; however, none were considered related to radial access.

Conclusion While femoral access remains the standard operating procedure for endovascular intervention of acute ischemic stroke, in patients with tortuous vascular anatomy, or having severe femoral disease, access via transfemoral is discouraged. Results from our study demonstrate that radial access is a safe and promising alternative, both as primary and rescue attempts. In an effort to reduce overall procedural time, radial access should be considered for qualified patients. A larger sample size would better validate the present findings.

Disclosures J. Farkas: None. N. Farkas: None. S. Feuerwerker: None. A. Tiwari: None. D. Turkel-Parrella: None. K. Arcot: None. K. Sivakumar: None.

\section{E-089 IATROGENIC POST-OPERATIVE CAROTID ARTERY PSEUDOANEURYSMS, DIAGNOSIS AND ENDOVASCULAR MANAGEMENT}

${ }^{1}$ E Nossek, ${ }^{2} \mathrm{~K}$ Prajoy, ${ }^{3} \mathrm{~J}$ Katz, ${ }^{4} \mathrm{~A}$ Setton. ${ }^{1}$ Neurosurgery, Maimonides Medical Center, New York, NY; ${ }^{2}$ ENT, North Shore University Hospital, Manhasset, NY; ${ }^{3}$ Neurology, North Shore University Hospital, Manhasset, NY; ${ }^{4}$ Neurosurgery and Radiology, North Shore University Hospital, Manhasset, NY

\subsection{6/neurintsurg-2016-012589.161}

Object Management of post-operative traumatic pseudoaneurysm of the external carotid and common carotid arteries has not been well characterized. Common presentation with early post-operative hemorrhages requires early diagnosis. We describe our experience with these lesions and review our diagnosis protocol, endovascular treatment and outcome.

Methods This is a retrospective review of patients treated between 2005-2014. Early post operative hemorrhages required immediate packing by ENT surgeon, hemodynamic stabilization and diagnostic workup, usually by CTA and DSA evaluation. We utilized an angiographic protocol to characterize the lesion, the vessel involved and the collateral circulation patency. We utilized combination of coils and nBCA glue embolization, to obliterate the lesion and the vessel segment along the external carotid distribution. Post embolization specific Super-selective injections were utilized to verify complete occlusion. Immediate and early clinical and angiographic results were reviewed.

Results We have treated 16 patients in this cohort. Thirteen lesions were associated with maxillofacial/oral surgeries and three were associated with tumor surgeries. Fifteen lesions were located in the ECA branches and one was located in the
CCA. Fifteen patients presented with acute hemorrhage. We identified 13 pseudoaneurysms and three vessels interruptions.

There were no procedural ruptures or complications. All aneurysms were completely obliterated, with early unpacking by ENT surgeon and hemodynamic stability, without any early re-hemorrhages. No early or delayed signs of ischemia were noted.

Conclusions Carotid artery pseudoaneurysm, post maxillofacial/ oral surgery can be treated early and effectively by endovascular procedures. High suspicion and early diagnosis associated with hemorrhages is crucial. Prompt imaging usually by CTA followed by selective cerebral angiography are highly effective. Utilization of coils and liquid embolic material can achieve immediate obliteration of the lesion and vessel segment. Selective post embolization studies of the collateral circulation are essential to confirm complete occlusion.

Disclosures E. Nossek: None. K. Prajoy: None. J. Katz: None. A. Setton: None.

\section{E-090 FEASIBILITY OF A VASCULAR REPLICATOR FOR ENDOVASCULAR PROCEDURE REHEARSAL}

C Nickele, D Hoit, L Elijovich, A Arthur. Neurosurgery, Semmes-Murphey Clinic, Memphis, TN

\subsection{6/neurintsurg-2016-012589.162}

Background Multiple studies have shown the benefit of simulator use for medical trainees. In the endovascular realm, this has been demonstrated in the cardiac, vascular and neurovascular literature with both computer simulation models and physical models of the vasculature. In this study, the authors investigate the feasibility of a customized 3D model, not for training purposes, but for rehearsal prior to an actual endovascular treatment procedure.

Methods Thirty-five patients with intracerebral aneurysms in various locations were enrolled in the study. Cases were chosen based on attending surgeon preference. Rotational 3D imaging studies of the pertinent vascular distribution were used to manufacture a 3D vascular model of that patient's vascular tree. Then, prior to the actual procedure, the attending neurointerventionalist performed a practice procedure on the $3 \mathrm{D}$ flow model. The model was used in conjunction with a complete system containing a biphasic pump to simulate the cardiac cycle and blood flow (Vascular Simulations, Stony Brook, USA). This was a full procedure, done as the treatment procedure would be, using research materials, and a complete neuroangiography system. After the actual treatment procedure, comments were recorded from the neurointerventionalist regarding the potential benefit of the practice session. Results Thirty-six procedures were done in thirty-five patients, 28 of the replicator sessions proved to be useful. Eight patients had replicator sessions that were not useful, and all of these were due to some failure of the replicated model. Models leaked in 4 cases, key vessels were not patent in the model in 4 cases, and 3 models had anatomic inaccuracies. When useful, the replicator sessions showed varying types of utility; allowing the operator to properly size a device, helping to choose the best wire and catheter combination to select a distal vessel, helping to understand the best landing zone for a stent, or simply allowing the operator to practice the procedure before the official treatment. 